\title{
Implementación de sensores de pH para valorar la conservación preventiva en un taller de restauración de pintura
}

Daniel Morales Martín, M. ${ }^{a}$ Ángeles Villegas Broncano | Instituto de Historia, Consejo Superior de Investigaciones Científicas (CSIC)

URL de la contribución <www.iaph.es/revistaph/index.php/revistaph/article/view/4608>

\section{RESUMEN}

La preservación del patrimonio cultural comprende la conservación preventiva, la conservación curativa y la restauración. La mayoría de estas tareas se realizan en talleres específicos donde los bienes residen durante periodos de tiempo variables. Puesto que en dichos espacios se utilizan reactivos químicos para múltiples trabajos, el control de la calidad del aire es importante para garantizar una óptima conservación de los objetos y unas condiciones de trabajo saludables. La evaluación del pH del aire es uno de los procedimientos más directos para conocer las posibilidades reales de degradación de los bienes culturales. Los sensores químicos patentados por el grupo de investigación CERVITRUM (Instituto de Historia, CSIC) pueden evaluar cualitativa y cuantitativamente el pH del aire. En esta investigación se utilizan con el objetivo de evaluar las condiciones ambientales en un taller de restauración de pintura sobre tela de la Facultad de Bellas Artes de la Universidad Complutense de Madrid. En el estudio se apunta la conveniencia de analizar la interacción del medio ambiente con los reactivos, tanto de forma individual como coordinada y en función de los espacios destinados a las tareas de restauración. Tras la síntesis, ubicación y registro de la respuesta de los sensores, los resultados indican que, en las condiciones habituales y con las variaciones climáticas producidas durante las evaluaciones, la calidad ambiental del taller es apta para garantizar la conservación de las obras durante su intervención.

\section{Palabras clave}

Ambiente | Conservación preventiva | pH | Pintura | Sensores | Taller de restauración | 

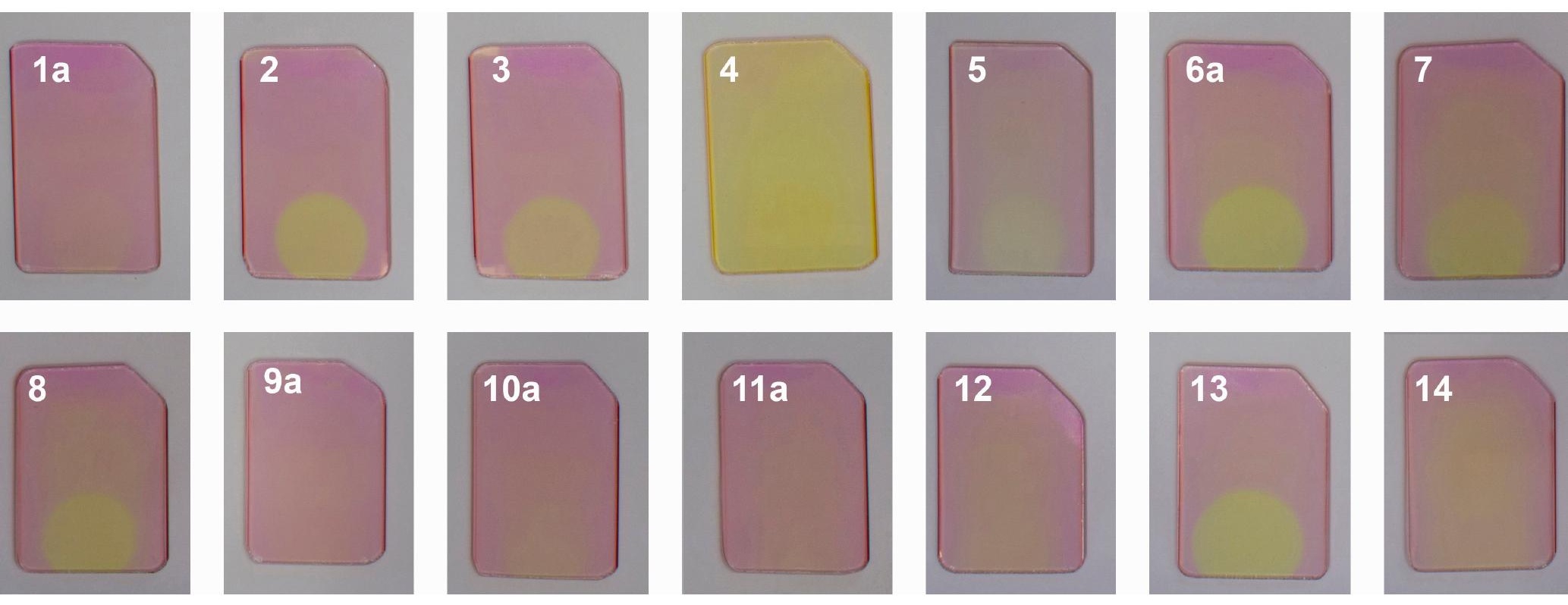

\section{Implementation of $\mathrm{pH}$ Sensors to assess preventive conservation in a paint restoration workshop}

\section{ABSTRACT}

The cultural heritage preservation brings together preventive conservation, remedial conservation and restoration. Most of these tasks are carried out in suitable workshops where cultural items are kept for variable periods of time. Since chemical reagents are used in such workshops for a wide variety of works, the air quality control is an important subject to guarantee the optimal conservation of the objects and to provide healthy working conditions, simultaneously. The assessment of the air $\mathrm{pH}$ is one of the most direct procedures to know the real degradation risk of cultural items. The chemical sensors patented by the research team CERVITRUM (Institute of History, CSIC) are able to qualitative and quantitatively evaluate the pH of the air. In the present research these sensors are used with the aim to evaluate the environmental conditions of a painting on canvas restoration workshop from the Fine Arts Faculty of the Complutense University (Madrid, Spain). The research scope highlights the importance of analyzing the interaction between the environment and the reagents, either individually or coordinately, and as a function of the spaces devoted to restoration tasks. After the synthesis, installation and recording of the sensors response, the results show that, under the normal use conditions and the climatic variations occurred during the evaluations carried out, the environmental quality of the workshop is suitable enough to guarantee the conservation of the paintings during the restoration process.

\section{Keywords}

Environment | Preventive conservation | pH | Painting | Sensors | Restoration Workshop |

Cómo citar: Morales Martín, D. y Villegas Broncano, Ma Á. (2021) Implementación de sensores de pH para valorar la conservación preventiva en un taller de restauración de pintura. revista $\mathrm{PH}, \mathrm{n} .{ }^{\circ}$ 102, pp. 98-116. Disponible en: www.iaph.es/revistaph/index.php/revistaph/article/view/4608 DOI 10.33349/2021.102.4608

Enviado: 20/03/2020 | Aceptado: 16/10/2020 | Publicado: 10/02/2021 


\section{INTRODUCCIÓN}

\section{Antecedentes de la evaluación ambiental mediante sensores de acidez}

Los sensores de $\mathrm{pH}$ ambiental y la unidad de medida portátil que se han utilizado en la presente investigación se patentaron en 2010 (Villegas Broncano et ál. 2010). Sin embargo, los trabajos experimentales previos que fueron necesarios para su caracterización químico-física, así como para el establecimiento de los procedimientos de calibración, se remontan a la década anterior (Carmona, Villegas y Fernández 2004).

En los primeros trabajos experimentales de evaluación ambiental los sensores se instalaron en el interior y exterior del Castillo de Wawel (Cracovia, Polonia) y en una estación meteorológica del centro urbano moderno de dicha ciudad. El método de medida de las respuestas de los sensores consistió en registrar los correspondientes espectros de absorción óptica en el laboratorio, una vez que los sensores se habían sensibilizado en sus respectivas posiciones (García-Heras et ál. 2005b; García-Heras et ál. 2005a). Este mismo método se llevó a cabo en la evaluación ambiental de los fondos documentales de la Biblioteca Tomás Navarro Tomás del Centro de Ciencias Humanas y Sociales del CSIC (Madrid), si bien en este caso se usó un espectrofotómetro portable que se desplazaba en un carro (Peña-Poza et ál. 2011a). Este fue el primer caso de estudio en el que la respuesta de los sensores se registró in situ. En la evaluación del pH ambiental que se llevó a cabo en el Palacio Real de Wilanów (Varsovia, Polonia), la respuesta de los sensores se registró también in situ gracias a un espectrofotómetro portable de pequeño tamaño (Peña-Poza et ál. 2011b).

El primer prototipo de unidad de medida portátil se probó durante las evaluaciones ambientales de la iglesia del Espíritu Santo y de la Sede Central del CSIC (Madrid) (Peña-Poza et ál. 2013). A este primer prototipo siguieron diseños más avanzados provistos de sondas de temperatura y de humedad relativa, que proporcionaban valores más precisos del $\mathrm{pH}$ ambiental y eliminaban las fluctuaciones que dichos parámetros pudieran tener sobre los componentes electrónicos del prototipo (Llorente-Alonso et ál. 2013a). La unidad de medida portátil definitiva se usó por primera vez en la evaluación ambiental del Museo del Ejército (Alcázar de Toledo) (Llorente-Alonso et ál. 2013b). En las evaluaciones ambientales del pH que se realizaron en la Fundación Centro Nacional del Vidrio, Real Fábrica de Cristales de La Granja (Segovia) y en parte del Museo Naval (Madrid) se aplicó la metodología recientemente optimizada gracias a un amplio trabajo de tesis doctoral (García-Heras y Villegas 2015; Peña-Poza et ál. 2015; Peña-Poza 2014). Dicha metodología consiste en lo siguiente: los sensores se exponen al ambiente a evaluar durante $24-48 \mathrm{~h}$ tras las cuales se registra su respuesta óptica; a continuación, se registran las respuestas al cabo de dos o tres días 
más y, por último, se realiza una medida de comprobación a los dos días siguientes. Con este protocolo una evaluación completa y fiable puede llevarse a cabo en una semana.

Por lo anteriormente expuesto la tecnología de los sensores de $\mathrm{pH}$ ambiental aplicada a la conservación preventiva del patrimonio cultural se considera un método innovador, preciso y fiable, como demuestran los casos de estudio citados. Una revisión de las características, propiedades y uso de estos sensores se puede consultar en la publicación Sol-Gel Environmental Sensors for Preventive Conservation of Cultural Heritage (Villegas, PeñaPoza y Garcia-Heras 2016).

\section{La conservación preventiva en los talleres de restauración}

La conservación preventiva queda principalmente sujeta al control de parámetros ambientales como la humedad relativa, la iluminación, la temperatura, el ruido y los contaminantes atmosféricos, entre otros, durante la exposición, la manipulación, el transporte, el almacenaje y los trabajos de restauración (García 2013, 19-23).

Tras una larga trayectoria, actualmente se cuenta con una serie de pautas específicas destinadas a la conservación preventiva de distintas obras en función de su naturaleza (Plan 2011). La pintura sobre lienzo cuenta con protocolos específicos para prevenir determinados riesgos y proteger las obras en diferentes espacios expositivos (Salas y Porras 2018, 85-88). Sin embargo, no se dispone de una bibliografía específica que abarque todas aquellas medidas de prevención a considerar en los talleres de restauración. Se ha de tener en cuenta que en dichos espacios las obras quedan expuestas en mayor magnitud a los factores susceptibles de alterar su estabilidad y, por ende, a su conservación: los agentes contaminantes derivados del almacenaje y la constante manipulación de productos químicos.

Desde el punto de vista de la conservación se entiende por contaminantes aquellos gases, líquidos y partículas sólidas en suspensión cuya presencia causa daños en las obras.

En un taller de restauración, al igual que en un museo u otro espacio de interés patrimonial, los contaminantes pueden proceder del exterior, al ventilar el espacio o por el trasiego de personal; y del interior, generados por el metabolismo humano, las emisiones del mobiliario, de los reactivos químicos almacenados y de las propias obras, así como de otros contaminantes derivados de ciertas actividades de restauración. De igual modo se cuenta con otra serie de sustancias contaminantes que no suponen un factor de alteración para las obras, pero que sí son perjudiciales para la salud humana (Grzywacz 2006). 
En las últimas décadas, con el fin de minimizar los riesgos para la salud de los conservadores-restauradores y en pro de la conservación de las obras pictóricas, se ha limitado el uso de disolventes de alta toxicidad en la práctica de la restauración (Sánchez 2012, 178-195). Estos se han sustituido por los llamados "disolventes verdes", además de modificar sus propiedades para disminuir la volatilidad del medio activo, lo que supone una mejora para la seguridad del restaurador (Salas y Porras 2018, 64-75). Las estrategias de control de contaminantes en museos y otros lugares contenedores de bienes culturales se basan en cinco pautas correlativas: evitar, bloquear, reducir, monitorizar y responder, y pueden igualmente aplicarse en los talleres de restauración (García 2013, 233-240). Estos cuentan, en la medida de lo posible, con los equipos necesarios para reducir o evitar la contaminación ambiental del espacio de trabajo y, así, garantizar en primer lugar la seguridad laboral, y en segundo lugar la conservación de las obras durante los procesos de restauración (García 2013, 213-233). Sin embargo, no todos los talleres cuentan con equipos especiales para extraer los gases contaminantes derivados de las limpiezas, del barnizado u otras actividades. Además, se ha de tener en cuenta el hecho de que en algunas ocasiones las intervenciones se realizan in situ, lo que dificulta o imposibilita la instalación de extractores de gases. Ante estas limitaciones, y de acuerdo a la última actualización del código de Prevención de Riesgos Laborales en España, la manipulación de productos químicos debe realizarse siempre con equipos de protección individual adecuados y en un espacio ventilado, siempre y cuando las emisiones gaseosas no dañen el medio natural (Prevención 2019, 112-128; 203-213).

\section{El taller de restauración de la Universidad Complutense de Madrid}

El aula-taller 011 de la Facultad de Bellas Artes de la Universidad Complutense de Madrid y las obras que en él se encuentran constituyen el objeto de estudio seleccionado para desarrollar esta investigación.

El espacio se localiza en el campus de Moncloa, distrito Moncloa-Aravaca, Madrid. El taller se encuentra en la planta cero del edificio principal de dicha facultad, construido en 1973 para acoger la Escuela de Bellas Artes, que, hasta entonces, compartía sede con la Academia de Bellas Artes en la Real Academia de Bellas Artes de San Fernando.

En un primer momento el taller fue la tienda de materiales para estudiantes. Posteriormente pasó a ser un aula de pintura, lo que llevó a la obligada instalación de un sistema de ventilación (extractores que se accionan automáticamente por la actividad del aire, colocados en la parte superior de algunas ventanas). Finalmente, y tras un tiempo desocupado, el aula pasó a ser el taller de restauración donde, desde el año 2009, se imparte la asignatura Metodología para la Conservación y Restauración de Pintura l. 


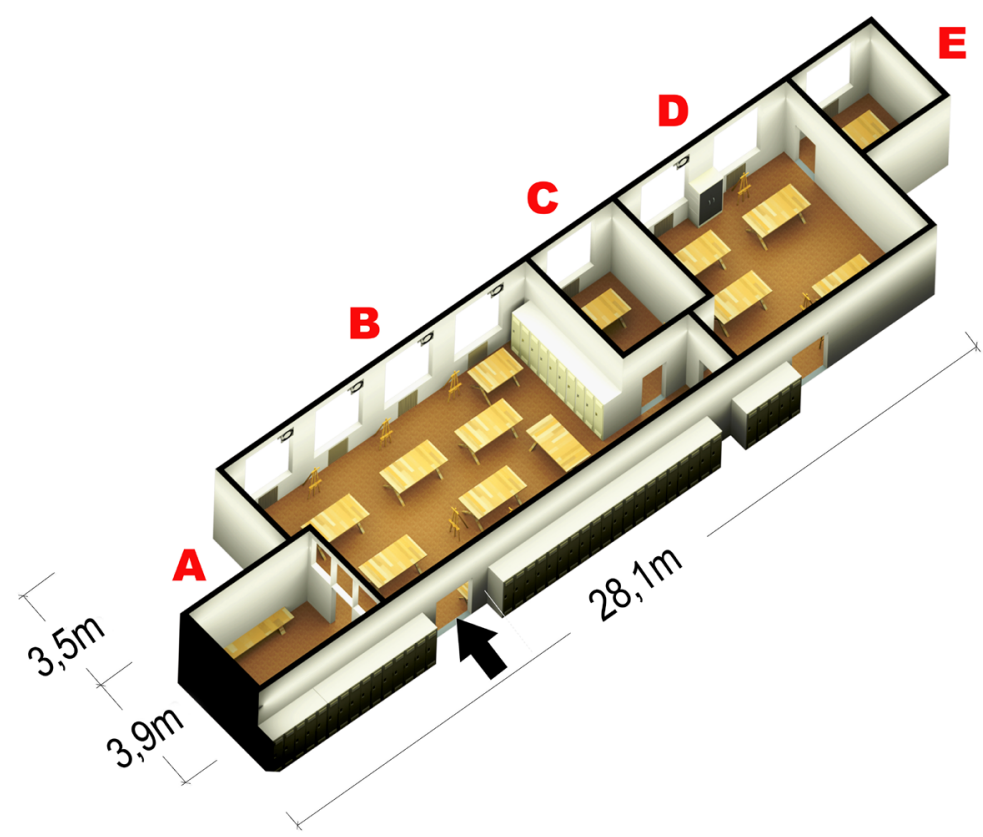

El taller, con orientación norte y un total de $805 \mathrm{~m}^{3}$, se compone de cinco salas (ver figura 1), de las cuales cuatro son exteriores, dando a un aparcamiento abierto en la calle Arquitecto López Otero. La primera sala (A) consiste en un cerramiento con paneles prefabricados que se utiliza como almacén de materiales auxiliares. La siguiente es una de las principales salas de trabajo (B) y por la que se accede al taller. Aquí se encuentran las pilas de lavado, una zona con hornillos y cuatro armarios de metal con materiales y productos específicos, entre ellos, disolventes y reactivos químicos. El despacho del profesorado (C) funciona como lugar de trabajo y custodia para las pinturas de mayor valor. Contiguo a éste se encuentra la segunda sala principal (D). Por último, se cuenta con una pequeña habitación destinada a realizar labores con piezas sumamente delicadas (E).

En cuanto a las características del taller cabe destacar, por un lado, el sistema de ventilación y, por otro, el régimen de climatización. El primero, descrito con anterioridad, solo se encuentra en las salas B y D. La segunda sala tiene un extractor de aire instalado en una de sus dos cristaleras, mientras que en la sala B hay uno por cada una de ellas. Asimismo, la sala A está comunicada con la B mediante una serie de ventanas ubicadas en la parte superior del panel principal. El taller no cuenta con un sistema de climatización específico. Este es de carácter general para toda la facultad, cuyo principal objetivo es el apropiado confort climático para el correcto desarrollo de las actividades del centro. De este modo la temperatura aproximada en los meses de otoño e invierno es de $21^{\circ} \mathrm{C}$ en todas las salas del taller, salvo en
Figura 1. Perspectiva con orientación norte del plano en tres dimensiones del aula-taller 011 infografía Yukisan López 
la sala A que carece de radiadores. En las estaciones de primavera y verano la temperatura media del aula oscila entre $25-28^{\circ} \mathrm{C}$. La humedad relativa del espacio de trabajo se encuentra en el intervalo de 30-35\%. La sala A, aislada del exterior y sin calefacción, registra unos valores de humedad relativa más bajos y estables (25-28 \%).

En el curso 2018/2019 hubo un total de 15 alumnos matriculados, organizados en grupos para intervenir durante dos días a la semana (jueves y viernes de 15:00 a 18:00 h) 13 de las 22 obras reunidas en el taller y distribuidas entre la sala B y D principalmente.

La importancia e innovación del presente trabajo reside en su carácter interdisciplinar y en la aplicabilidad de los resultados obtenidos, que permiten ajustar las condiciones de climatización y/o ventilación del espacio analizado, así como tomar decisiones relativas a la conservación preventiva de forma anticipada. El objetivo principal de esta investigación ha sido llevar a cabo la evaluación de la calidad ambiental de un taller de restauración de pintura sobre lienzo mediante la evaluación del pH del aire con sensores ópticos sol-gel, así como detectar eventos debidos a la manipulación de reactivos químicos, cambios climáticos, trasiego de personal y régimen de ventilación a través del estudio de la respuesta de los sensores de $\mathrm{pH}$ ambiental tras su instalación y sensibilización.

\section{METODOLOGÍA}

\section{Síntesis de los sensores}

La elaboración de los sensores de pH ambiental se ha llevado a cabo de acuerdo a su patente de invención (Villegas Broncano et ál. 2010). Los sensores de acidez ambiental se basan en la tecnología sol-gel cuya preparación parte de una lámina de vidrio de silicato sódico cálcico, que actúa como soporte de un recubrimiento sensible. Esta película se obtiene a partir de una suspensión coloidal (sol) a la que se le incorpora un colorante orgánico que actúa como fase sensible, rojo de clorofenol. La aplicación del sol en el soporte se realiza por el método de inmersión-extracción, con el que se obtiene una película de $250-450 \mathrm{~nm}$ de espesor por ambas caras del soporte. Durante este procedimiento de deposición se generan dos defectos en el recubrimiento recién aplicado: defecto de frente (marca del nivel del sol) y defecto de gota (acumulación del sol en la parte inferior del sustrato de vidrio). Ambas irregularidades se eliminan por corte una vez concluida la estabilización físicoquímica del recubrimiento, a la que se llega mediante el proceso de densificación parcial (gel) del mismo. Esta densificación se alcanza con un tratamiento térmico a $60^{\circ} \mathrm{C}$ durante 72 horas en una estufa de aire forzado. El resultado final es una fina capa coloreada de 
gel en ambas caras del vidrio, al que se le da el tamaño y la forma definitiva mediante corte y pulido, obteniéndose así el sensor propiamente dicho. Para el estudio realizado se sintetizaron un total de 20 sensores.

\section{Validación de los sensores}

En la figura 2 se han representado los espectros visibles de los sensores para tres valores representativos de la escala de $\mathrm{pH}$ (ácido, neutro y básico), lo que indica cualitativamente la sensibilidad óptica de los mismos y dónde se sitúan los máximos de absorción visible para esos valores de pH.

Previamente a la instalación de los sensores en el espacio de estudio seleccionado se ha de conocer su respuesta óptica cuantitativa, es decir, las variaciones de la intensidad de la absorción de los sensores en el intervalo de la longitud de onda de la radiación visible en función del pH. Para ello, se siguió el protocolo de calidad previamente establecido en el que se estudia la absorción visible del sensor a pH 5 y 7 . Una vez que los sensores se exponen a dichos $\mathrm{pH}$ se registra el correspondiente espectro visible (intervalo de 380 a $750 \mathrm{~nm}$ ), con la unidad de medida portátil (Villegas Broncano et ál. 2010), y se registra la intensidad del máximo característico de la banda de absorción a $\lambda=575,44 \mathrm{~nm}$, que se corresponde con la absorción característica de la forma tautomérica básica de la especie sensible inmovilizada en el recubrimiento sol-gel. Una vez registrada la respuesta de todos los sensores se estudia el promedio de absorción a ambos pH, su desviación respecto a la media global de todos los sensores y la desviación entre las medidas de un mismo sensor para cada pH (parámetro $\sigma$ ). Este método per-

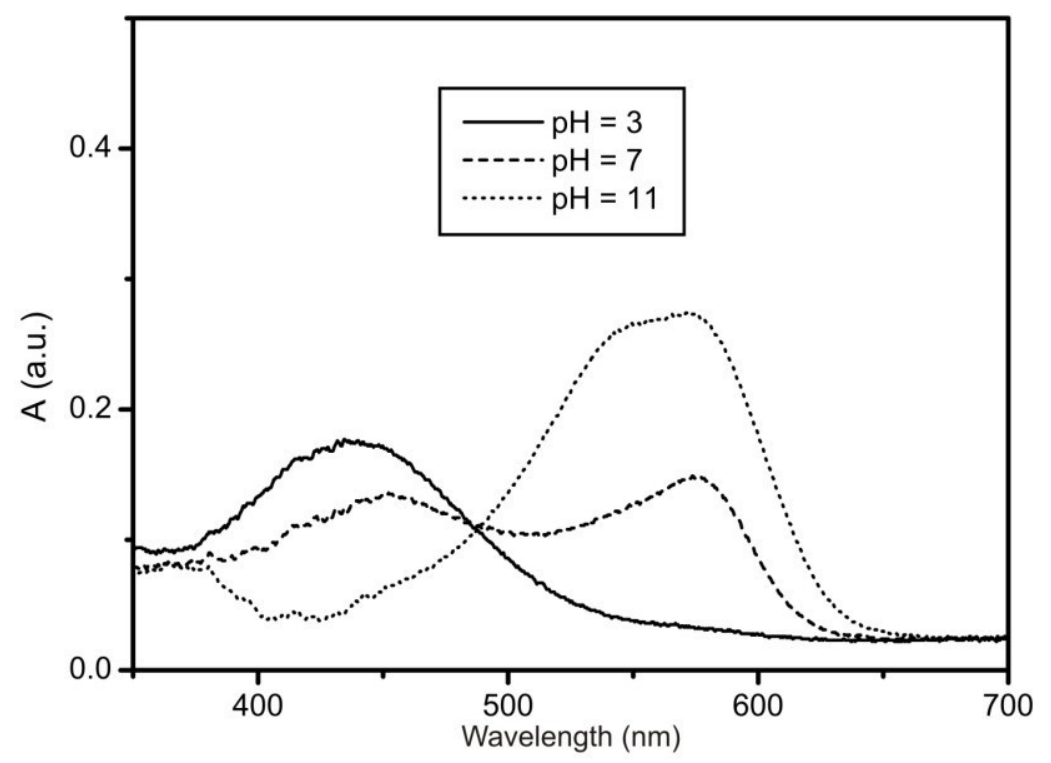

Figura 2. Espectros de absorción visible del sensor a pH ácido, neutro y básico | gráfico grupo de investigación CERVITRUM 
mite clasificar la calidad de los sensores, así como descartar todos aquellos cuya respuesta se desvía más de 0,2 unidades de la escala de pH respecto al pH correspondiente al promedio calculado para la intensidad de absorción a 575,44 nm. También se desechan los sensores cuya respuesta se aleja considerablemente de la desviación típica calculada para los valores de un $\mathrm{pH}$ determinado. De acuerdo con estos criterios, se establece la siguiente clasificación alfabética: A es una calidad óptima, B y C es una calidad aceptable, y D se considera inapropiada, es decir, son aquellos sensores que se desechan. Dentro del primer grupo se encuentra la calidad A+, que corresponde a los sensores de calidad óptima y que se utilizan para la siguiente fase: la calibración.

Los resultados de calidad obtenidos tras aplicar el método de validación en los sensores sintetizados son los siguientes:

A+: 3 sensores ( $n .{ }^{0} 1,9$ y 12$), 15 \%$

A: 10 sensores (n. ${ }^{\circ} 2,3,4,6,8,11,14,16,17$ y 18$), 50 \%$

B: 5 sensores ( $\left(.^{\circ} 5,7,10,15\right.$ y 20), $25 \%$

C: 2 sensores ( ${ }^{\circ} 13$ y 19$), 10 \%$

\section{Calibración de los sensores}

La calibración se realiza a partir de los sensores A+ que se han obtenido, ya que su respuesta óptica tiene la menor desviación posible respecto al pH promedio y a la desviación típica entre sus mismas respuestas. El procedimiento de calibración se desarrolla siguiendo una metodología semejante a la del proceso de validación. Sin embargo, en esta fase se trabaja con cuatro soluciones tampón a pH 5, 6, 7 y 8. Estos valores de pH abarcan las condi-

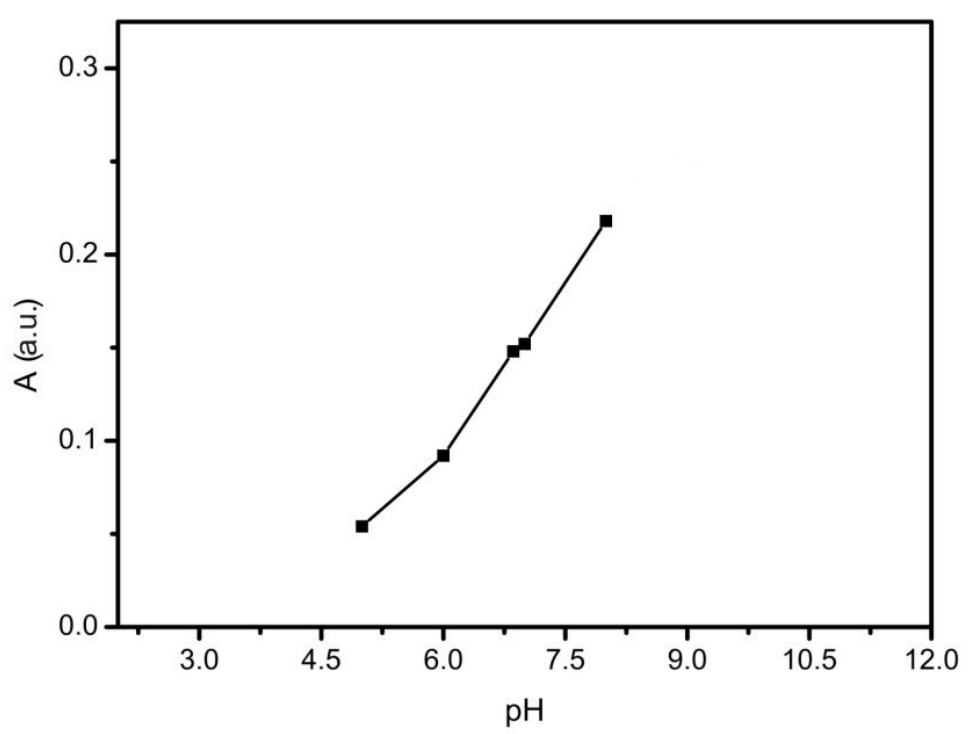


ciones en las que, de forma general, conviene controlar la alteración de los materiales artísticos, y, por lo tanto, constituyen el intervalo de $\mathrm{pH}$ en el que interesa focalizar el estudio. Asimismo, estos valores de $\mathrm{pH}$ van a constituir los puntos experimentales de la curva de calibración, donde una vez registrada la respuesta de los sensores para cada $\mathrm{pH}$ se calcula el promedio de las medidas realizadas y su resultado se considera el valor de absorción característico para cada $\mathrm{pH}$. A continuación, se realiza el ajuste lineal para cada intervalo de $\mathrm{pH}$ obteniendo una precisión general de 0,1 unidades de la escala de $\mathrm{pH}$ (ver figura 3 página anterior). Finalmente, esta información se incorpora al software de la unidad de medida portátil para directamente determinar el pH en función de la absorción visible de los sensores tras su exposición en el espacio a estudiar.

\section{Acondicionamiento previo de los sensores}

Con el objetivo de optimizar la respuesta de los sensores se llevó a cabo una labor de acondicionamiento previo a su ubicación en el taller. Esta tarea consiste en la inmersión de los sensores en una solución tampón de pH neutro $(7,0)$ durante $10 \mathrm{~min}$. Para evitar la sensibilización de los sensores durante su traslado desde el Laboratorio de Conservación del Patrimonio del Instituto de Historia a la Facultad de Bellas Artes de la Universidad Complutense de Madrid, se introdujeron en bolsas de polietileno con cierre hermético.

\section{Instalación y registro de la respuesta óptica de los sensores}

Tanto la instalación como la posterior medida de la respuesta de los sensores se vio supeditada al calendario académico de la Facultad de Bellas Artes para el curso 2018/2019, así como a la planificación interna de la asignatura Metodología para la Conservación y Restauración de Pintura (I) impartida por la doctora Alicia Sánchez Ortiz en el taller los jueves y viernes de 15:00 a 18:00 h. De este modo se organizaron un total de cinco evaluaciones, distribuidas entre el mes de noviembre de 2018 y el mes de junio de 2019. A la hora de organizar el inicio de cada evaluación se tuvo en cuenta la metodología específica desarrollada para tal fin. Según esta, la primera medida de los sensores ha de realizarse pasadas 24 horas desde su exposición, seguida de una segunda medida a las 48 horas y una tercera a las 72 horas, efectuando las siguientes en periodos más prolongados (2-3 días) si no se observan cambios notables. Se da por finalizada una evaluación cuando los valores de $\mathrm{pH}$ se estabilizan, lo que sucede como muy tarde a los 14-20 días sin contar con ningún tipo de incidente que pueda modificar las condiciones de acidez ambiental. De esta manera el periodo de cada evaluación fue el siguiente:

1. ${ }^{a}$ Evaluación del 19 de noviembre al 5 de diciembre de 2018 (15 días)

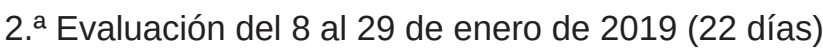


Tabla 1. Relación de las posiciones de los sensores en las distintas evaluaciones
3. ${ }^{a}$ Evaluación del 19 de febrero al 4 de marzo de 2019 (15 días)

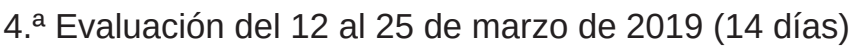

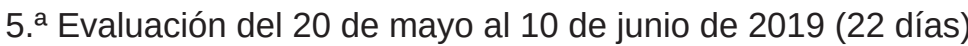

La distribución, posición y número de sensores en el espacio se realizó en función de los intereses de cada una de las cinco salas que conforman el taller (ver tabla 1). En cada evaluación se cambió la ubicación de algunos sensores con el objetivo de conocer la homogeneidad del pH ambiental. Asimismo, se incorporaron más sensores en aquellos espacios en los que, debido a los trabajos que se estaban realizando, se consideró necesario.

La colocación de los sensores en el taller se realizó mediante el uso de fundas de polietileno con una perforación doble en la parte inferior, dotadas de una pinza colgante para su sujeción. El registro de los resultados se rea-

\begin{tabular}{|c|c|c|c|}
\hline Posición & N. ${ }^{\circ}$ sensor & Evaluación & Descripción \\
\hline 1 & \multirow{2}{*}{2} & $1 .^{\mathrm{a}}, 2 .^{\mathrm{a}}$ y $3 .^{\mathrm{a}}$ & Colgado en la pared frente a la puerta de entrada (sala A) \\
\hline $1 \mathbf{a}$ & & $4 .^{a}$ y $5 .^{a}$ & Colgado cerca de la puerta de entrada (sala A) \\
\hline 2 & 3 & $1 .^{\mathrm{a}}, 2 .^{\mathrm{a}}, 3 .^{\mathrm{a}}, 4 .^{\mathrm{a}}$ y $5 .^{\mathrm{a}}$ & Sobre las pilas de lavado, próximo a la ventana (sala B) \\
\hline 3 & 4 & $1 .^{\mathrm{a}}, 2 .^{\mathrm{a}}, 3 .^{\mathrm{a}}, 4 .^{\mathrm{a}}$ y $5 .^{\mathrm{a}}$ & En la estantería de útiles de trabajo, próximo a los fogones (sala B) \\
\hline 4 & 6 & $1 .^{\mathrm{a}}, 2 .^{\mathrm{a}}, 3 .^{\mathrm{a}}, 4 .^{\mathrm{a}}$ y $5 .^{\mathrm{a}}$ & En el interior del armario de disolventes y reactivos químicos (sala B) \\
\hline 5 & 8 & $1 .^{\mathrm{a}}, 2 .^{\mathrm{a}}, 3 .^{\mathrm{a}}, 4 .^{\mathrm{a}}$ y $5 .^{\mathrm{a}}$ & Próximo a las ventanas y a los armarios de materiales (sala B) \\
\hline 6 & \multirow{2}{*}{5} & $2 .^{a}$ & Sobre los armarios de materiales (sala B) \\
\hline $6 \mathbf{a}$ & & $3 .^{a}, 4 .^{a}$ y $5 .^{a}$ & Encima del armario de disolventes y reactivos químicos (sala B) \\
\hline 7 & 10 & $4 .^{\mathrm{a}}$ y $5 .^{\mathrm{a}}$ & En la antesala entre las salas B y D (sala B) \\
\hline 8 & 15 & 4. ${ }^{\mathrm{a}}$ y $5 .^{\mathrm{a}}$ & Colgado próximo a la puerta de entrada al aula (sala B) \\
\hline 9 & \multirow[t]{2}{*}{11} & $1 .^{\mathrm{a}}, 2 .^{\mathrm{a}}$ y $3 .^{\mathrm{a}}$ & Colgado en un cartel, próximo a la entrada (sala C) \\
\hline $9 a$ & & 4. ${ }^{\mathrm{a}}$ y $5 .^{\mathrm{a}}$ & Colgado en un cartel, próximo a la ventana (sala C) \\
\hline 10 & \multirow{2}{*}{14} & 1. ${ }^{\mathrm{a}}$ y $2 .^{\mathrm{a}}$ & Colgado de un cartel, próximo a la ventana (sala D) \\
\hline $10 \mathrm{a}$ & & 3. ${ }^{a}, 4 .^{a}$ y $5 .^{a}$ & Colgado de un cartel, próximo a la ventana (sala D) \\
\hline 11 & \multirow{2}{*}{7} & $2 .^{a}$ y $3 .^{a}$ & Colgado próximo a la puerta de entrada (sala D) \\
\hline $11 \mathrm{a}$ & & $4 .^{\mathrm{a}}$ y $5 .^{\mathrm{a}}$ & Colgado en la pared del fondo (sala D) \\
\hline 12 & 16 & $1 .^{\mathrm{a}}, 2 .^{\mathrm{a}}, 3 .^{\mathrm{a}}, 4 .^{\mathrm{a}}$ y $5 .^{\mathrm{a}}$ & Colgado al fondo de la sala (sala E) \\
\hline 13 & 17 & $1 .^{\mathrm{a}}, 2 .^{\mathrm{a}}, 3 .^{\mathrm{a}}, 4 .^{\mathrm{a}}$ y $5 .^{\mathrm{a}}$ & En el exterior del taller, en la calle que da al aparcamiento abierto (exterior de la facultad) \\
\hline 14 & 18 & $1 .^{\mathrm{a}}, 2 .^{\mathrm{a}}, 3 \cdot^{\mathrm{a}}, 4 .^{\mathrm{a}}$ y $5 .^{\mathrm{a}}$ & En el exterior del taller, en el pasillo de taquillas que da acceso al taller (interior de la facultad) \\
\hline
\end{tabular}


lizó in situ (ver figura 4) gracias a la unidad de medida portátil, la misma empleada anteriormente para la validación y calibración de los sensores.

Una vez registrada la respuesta de los sensores, éstos se devolvieron a sus mismas posiciones. Finalizada una evaluación completa se procede a la recalibración de los sensores. Esta tarea consiste en repetir el procedimiento de calibración con los sensores que ya se han expuesto y sensibilizado, con el fin de regenerarlos y poderlos reusar con garantías de precisión.

\section{RESULTADOS Y DISCUSIÓN}

Con los resultados obtenidos tras registrar durante seis meses la respuesta óptica de los sensores de $\mathrm{pH}$ en unas determinadas condiciones ambientales, se ha valorado el pH promedio, el pH máximo, el pH mínimo y el parámetro $\Delta \mathrm{pH}$ (diferencia entre el $\mathrm{pH}$ máximo y mínimo registrados por cada sensor en cada evaluación). Con el análisis de estos parámetros se determinan las variaciones de acidez ambiental y el posible riesgo que, directamente, supone para las pinturas y el personal que se encuentran en el taller.

En la primera evaluación se ha obtenido un valor promedio de carácter neutro en la escala de $\mathrm{pH}$, con un máximo de $\mathrm{pH}=7,2$ en la sala $\mathrm{B}$ y un mínimo de $\mathrm{pH}=6,9$ en la sala $\mathrm{E}$. El sensor que ocupa la posición 13, expuesto a la intemperie, ha registrado un valor ácido $(\mathrm{pH}=6,1)$ debido a la contaminación ambiental urbana (ver figura 5, p. 110).

Igualmente, en los resultados de la segunda evaluación se han obtenido valores medios de $\mathrm{pH}$ neutro, comprendidos entre 7,2 y 6,9 en todas las salas del aula. La posición 13 correspondiente al sensor colocado en el exterior ha registrado un mínimo de pH 6,6 debido a la contaminación ambiental urbana. En la figura 6 (p. 110) se observan una serie de variaciones respecto a los resultados de la primera evaluación.

Este hecho se debe al inicio de actividades con disolventes orgánicos y productos químicos en el taller para acometer las intervenciones de restauración de las superficies pictóricas. A diferencia de los trabajos desarrollados durante la primera evaluación, las actividades llevadas a cabo durante la segunda precisan de una mayor ventilación del espacio. Esta ventilación se realiza con la apertura de ventanas, lo que se ve reflejado en las respuestas de los sensores de las posiciones 2 y 5 . Además, el sensor de la posición 4 ubicado en el interior del armario de disolventes ha registrado un valor de pH ácido, con un mínimo de 6,7, lo que se atribuye a la manipulación de los contenedores de reactivos y otros productos que estaban cerrados desde la clausura del curso anterior en el mes de mayo de 2018.

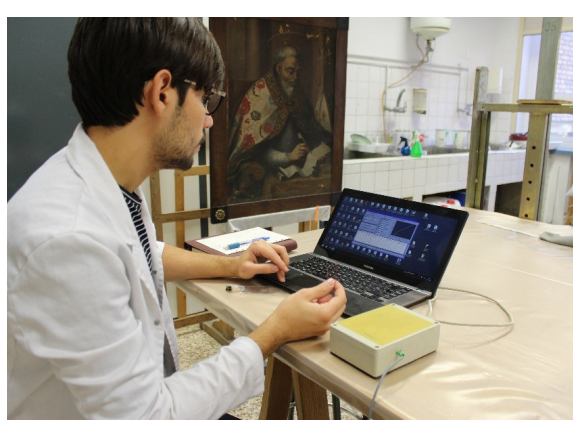

Figura 4. Registro in situ, en el taller de Conservación-Restauración de Pintura de la Facultad de Bellas Artes de la Universidad Complutense de Madrid, de la respuesta de los sensores de $\mathrm{pH}$ con la unidad de medida portátil 
Figura 5. Valores promedio, máximos y mínimos registrados por los sensores ópticos de $\mathrm{pH}$ en las diferentes salas del taller de restauración durante la primera evaluación
Figura. 6. Valores promedio, máximos y mínimos registrados por los sensores ópticos de $\mathrm{pH}$ en las diferentes salas del taller de restauración durante la segunda evaluación
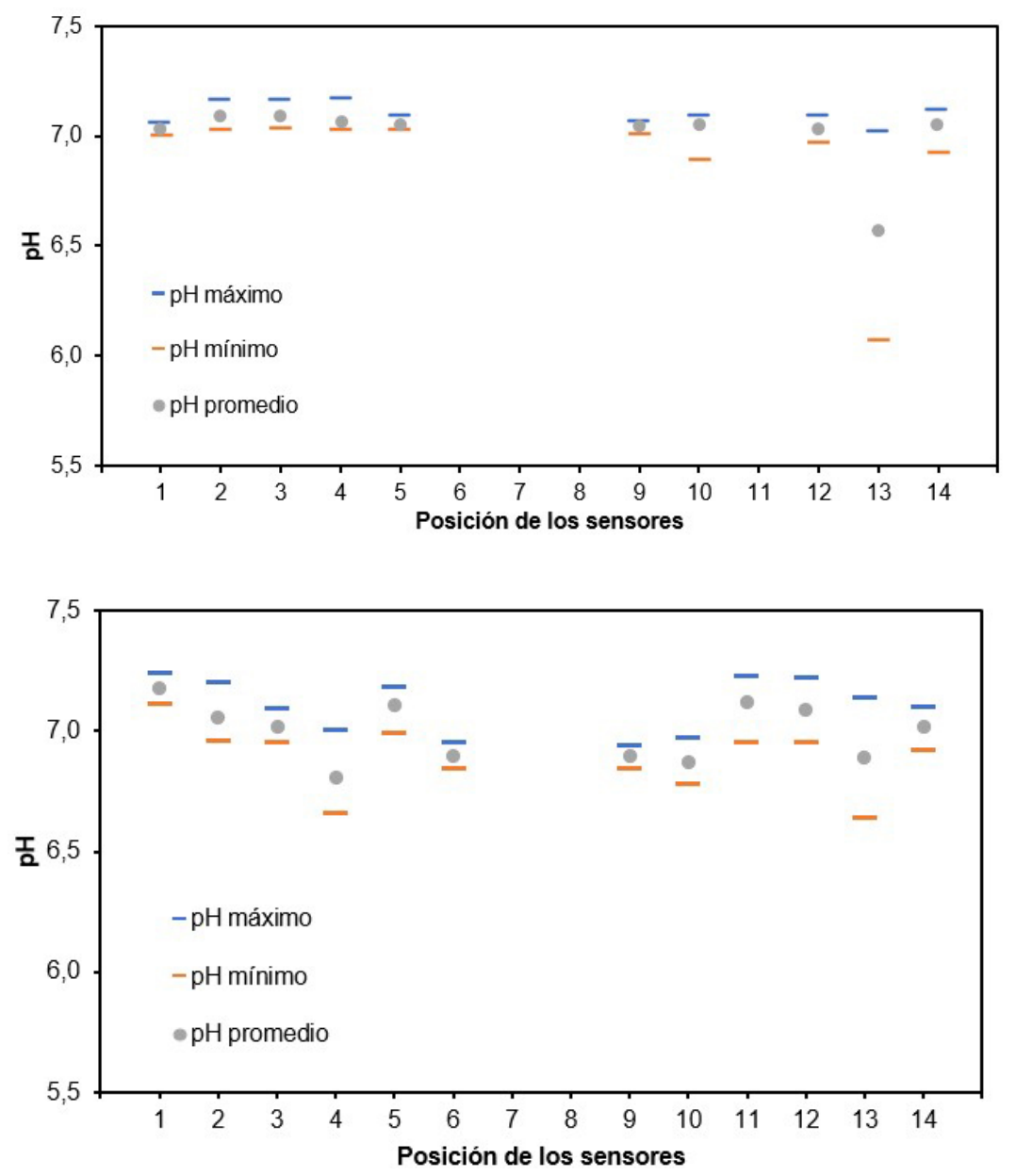

En la tercera evaluación se obtuvo, un promedio de pH neutro en el interior del taller, mientras que en el exterior se llegó a registrar un valor mínimo de 5,7 en la escala de $\mathrm{pH}$ (ver figura 7 ). Sin embargo, el viernes día 1 de marzo se prepararon en la sala B del aula una serie de soluciones acuosas para proceder a la limpieza superficial de una obra. Esta actividad supuso la alteración de la acidez ambiental hasta el momento analizada en dicha sala. La preparación de este medio de limpieza requiere la utilización de diferentes sales y ácidos (Cremonesi 2011) almacenados en los armarios del taller salvo uno, el ácido clorhídrico, hasta entonces almacenado en otro taller. La elaboración de las soluciones, la cual, y debido a la ausencia de campanas extractoras de gases, se realizó en una mesa próxima a las ventanas. El mismo día al finalizar la clase y proceder al registro de la respuesta de los sensores con la unidad de medida portátil se detectó, respecto al valor registrado dos días antes $(\mathrm{pH}=6,8 ; \mathrm{pH}=7,1)$, una variación de $\mathrm{pH}$ de 0,1 y 0,2 en los sensores $5(\mathrm{pH}=6,7)$ y $6 \mathrm{a}(\mathrm{pH}=6,9)$, respectivamente. Ambos senso- 

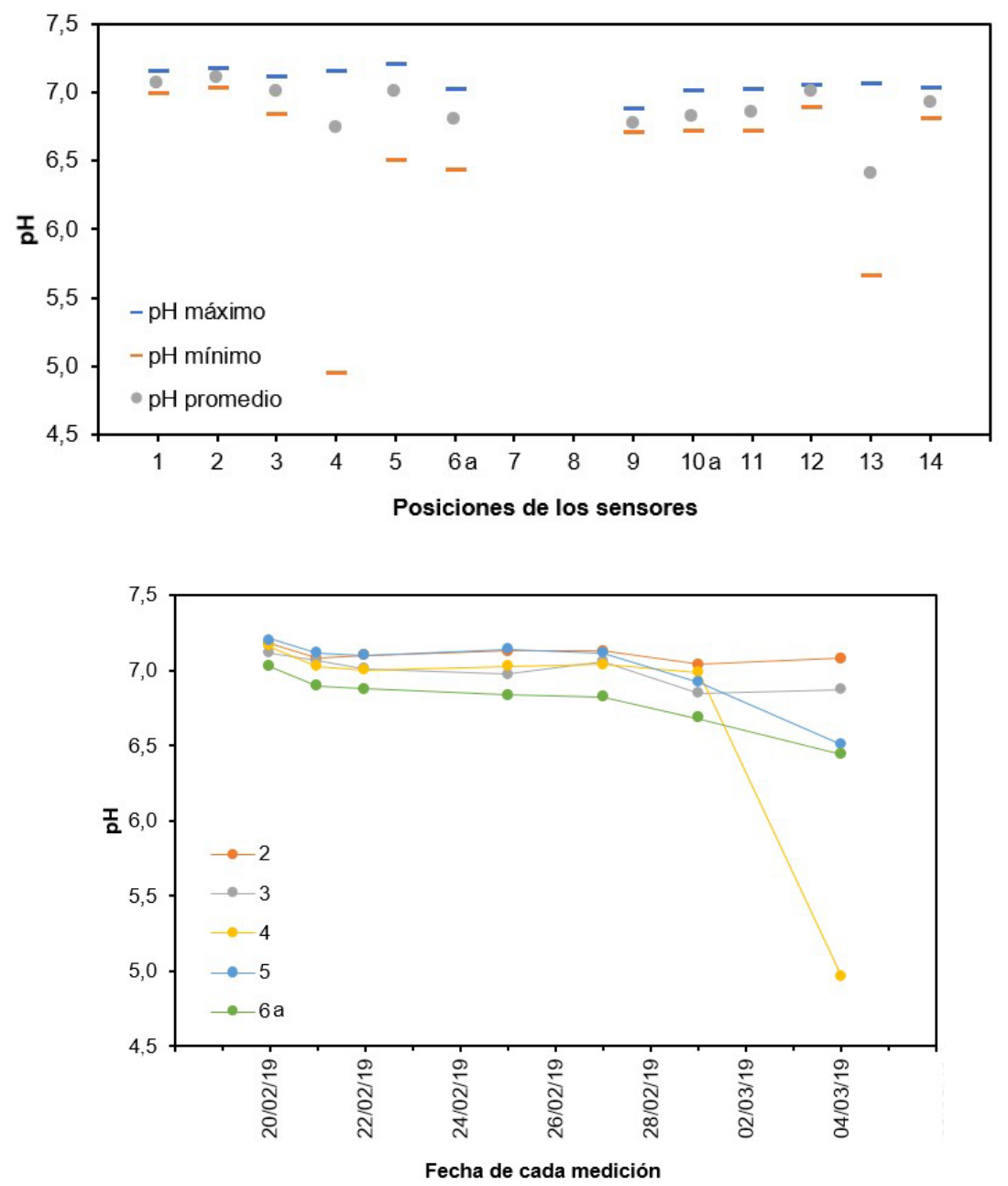

res ocupan una posición próxima a la zona de trabajo donde se manipuló el ácido clorhídrico. Después de 72 horas, en el último registro previsto para esta tercera evaluación, se comprobó cualitativa y cuantitativamente la acidificación del $\mathrm{pH}$ ambiental en aquellas posiciones donde se había manipulado el ácido clorhídrico. El sensor colocado en el interior del armario de disolventes y reactivos (posición 4), donde actualmente se almacena el ácido clorhídrico, registró un $\mathrm{pH}$ de 5,0. Asimismo, los sensores 5 y 6 a dieron un valor de $\mathrm{pH}$ ambiental ligeramente ácido (6,5 y 6,4, respectivamente) (ver figura 8). Este fenómeno se debe a la alta volatilidad de dicho ácido (presión de vapor: $20 \mathrm{hPa}$ ) y a la escasa ventilación del taller al permanecer cerrado durante el fin de semana.

En la cuarta evaluación todos los sensores registraron, cualitativa (ver figura 9, p. 112) y cuantitativamente (figura 10, p. 112), un pH ambiental ácido con un promedio de 6,1 en el exterior y 6,6 en el interior de la facultad; y en las
Figura 7. Valores promedio, máximos y mínimos registrados por los sensores ópticos de $\mathrm{pH}$ en las diferentes salas del taller de restauración durante la tercera evaluación
Figura 8. Representación de la evolución del pH en función de las fechas de registro de algunos sensores correspondientes al conjunto de la tercera evaluación en la sala B del taller

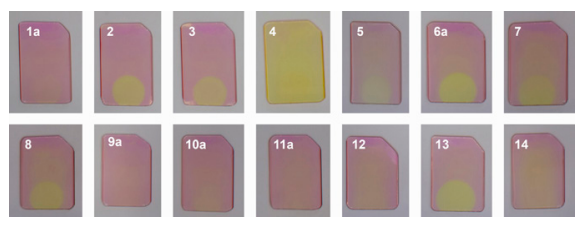

Figura 9. Aspecto de los sensores tras finalizar la cuarta evaluación 
Figura 10. Valores promedio, máximos y mínimos registrados por los sensores ópticos de $\mathrm{pH}$ en las diferentes salas del taller de restauración durante la cuarta evaluación
Figura 11. Valores promedio, máximos y mínimos registrados por los sensores ópticos de $\mathrm{pH}$ en las diferentes salas del taller de restauración durante la quinta evaluación diferentes salas del taller un valor medio comprendido entre 6,7 y 6,1 en la escala de $\mathrm{pH}$ (ver figura 10). Estos resultados se deben a un fenómeno macroclimático producido en la Península Ibérica como consecuencia de las escasas precipitaciones registradas durante el mes de marzo de 2019 , lo que supuso un aumento de la contaminación atmosférica que puede desembocar en la denominada deposición ácida seca.

En la Comunidad de Madrid, con dos únicos días de lluvia (4 y 6 de marzo) y $13 \mathrm{~mm}$ de agua recogidos (Medina 2019), se duplicaron los niveles de contaminación. Este evento climático ha quedado patente en todos los sensores instalados para el presente estudio, incluido el sensor colocado en el pasillo de taquillas y aislado directamente del exterior. La respuesta de este último sensor indica que el interior de la facultad también se vio afectado por este fenómeno de deposición ácida seca.
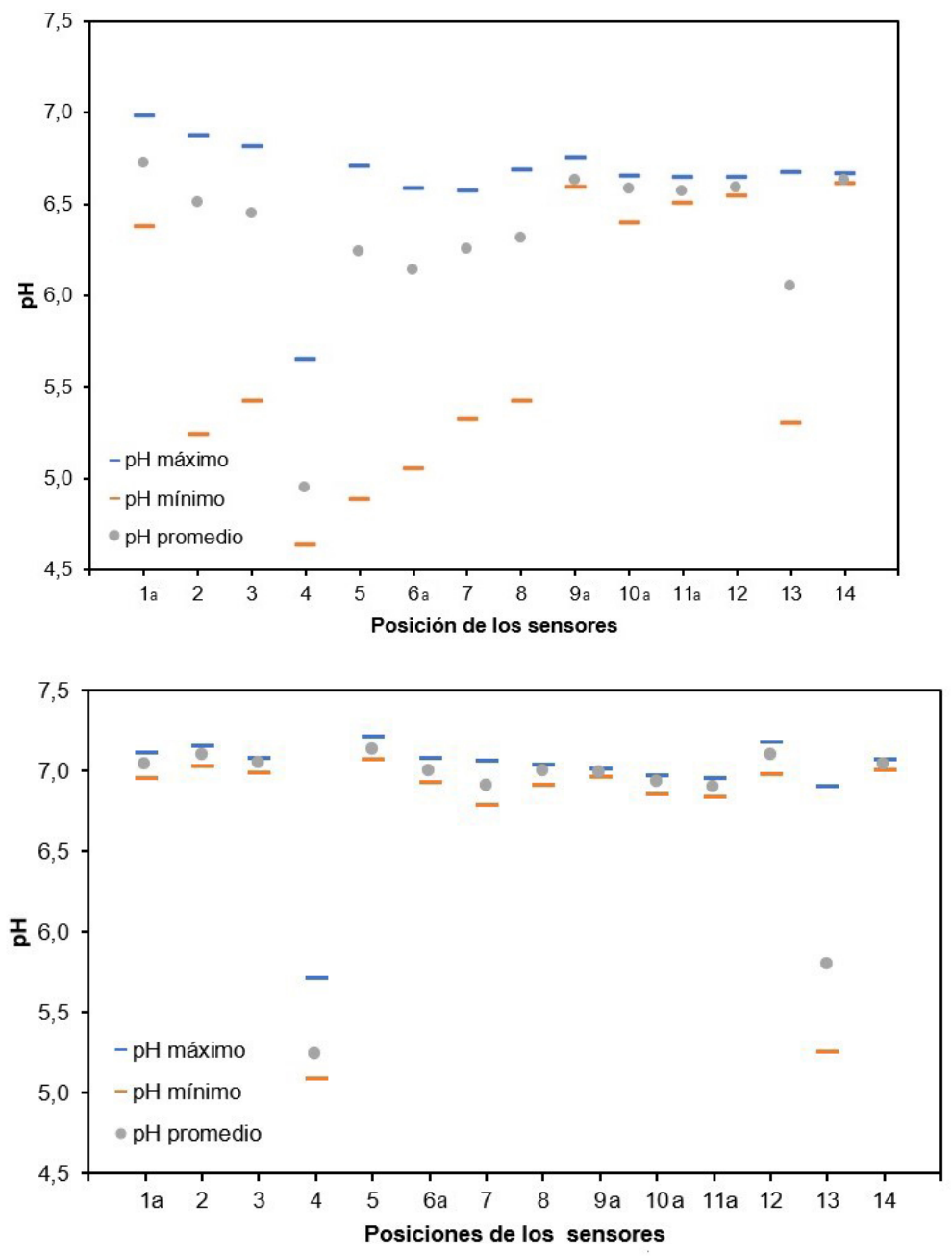
Finalmente, en la quinta y última evaluación se han registrado unos valores de $\mathrm{pH}$ neutros, a excepción del exterior $(\mathrm{pH}=5,8)$ y el armario de materiales que sigue custodiando el ácido clorhídrico ( $\mathrm{pH}=5,2)$ (ver figura 11). Con el fin de evaluar el riesgo que las condiciones ambientales del taller puedan tener en la correcta conservación de su contenido y personal, a lo largo de cada evaluación se han realizado una serie de gráficas donde solo se han tenido en cuenta los resultados obtenidos en el interior del taller. Según el criterio desarrollado por los inventores de esta metodología de evaluación ambiental, se han relacionado los diferentes parámetros anteriormente registrados y analizados, y se ha obtenido una clasificación de nivel de riesgo bajo, leve, medio y alto (ver tabla 2, p. 114).

Las figuras 12, 13 y 14 muestran cómo en condiciones normales la acidez ambiental del taller y sus fluctuaciones no suponen un riesgo para la conser-

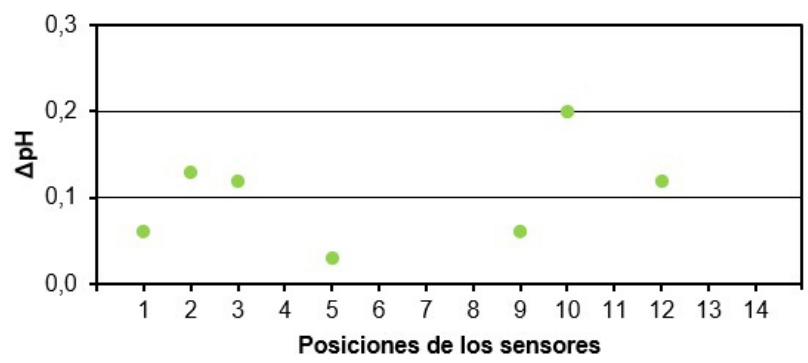

Nivel de riesgo 1

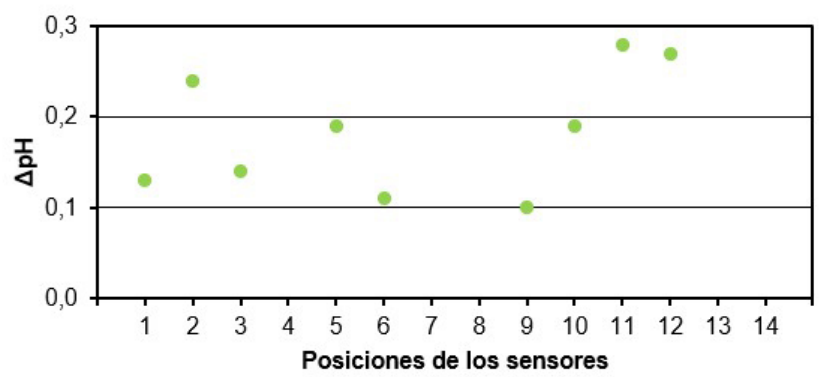

Nivel de riesgo 1

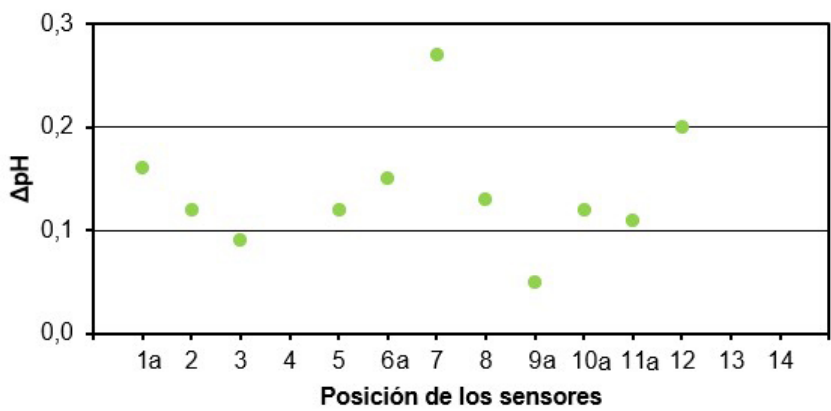

Figura 12. Resultados de la variación del pH ambiental de cada sensor instalado en el interior del aula durante la primera evaluación

Figura 13. Resultados de la variación del pH ambiental de cada sensor instalado en el interior del aula durante la segunda evaluación

- Nivel de riesgo 1

Figura 14. Resultados de la variación del pH ambiental de cada sensor instalado en el interior del aula durante la quinta evaluación 


\begin{tabular}{|c|c|c|c|}
\hline Color asignado & $\begin{array}{l}\text { Nivel de riesgo de choque } \\
\text { ácido }\end{array}$ & pH promedio & $\Delta \mathrm{pH}$ \\
\hline Verde & 1 (bajo) & $\mathrm{pH}$ promedio $>6,5$ & $\Delta \mathrm{pH}<0,8$ \\
\hline Amarillo & 2 (leve) & $\mathrm{pH}$ promedio $>6,5$ & $\Delta \mathrm{pH}>0,8$ \\
\hline Naranja & 3 (medio) & $\mathrm{pH}$ promedio $<6,5$ & $\Delta \mathrm{pH}<0,8$ \\
\hline Rojo & 4 (alto) & $\mathrm{pH}$ promedio $<6,5$ & $\Delta \mathrm{pH}>0,8$ \\
\hline
\end{tabular}

Tabla 2. Relación entre el valor promedio de $\mathrm{pH}$, el parámetro $\Delta \mathrm{pH}$ y el riesgo de choque ácido (Peña Poza 2014)

Figura 15. Resultados de la variación del pH ambiental de cada sensor instalado en el interior del aula durante la tercera evaluación (Fuente: los autores)

Figura 16. Resultados de la variación del pH ambiental de cada sensor instalado en el interior del aula durante la cuarta evaluación (Fuente: los autores)

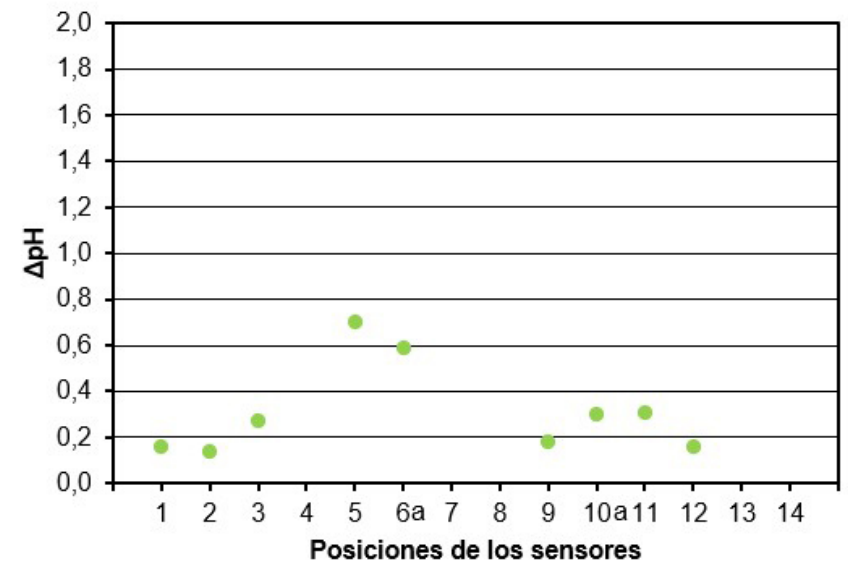

- Nivel de riesgo 1

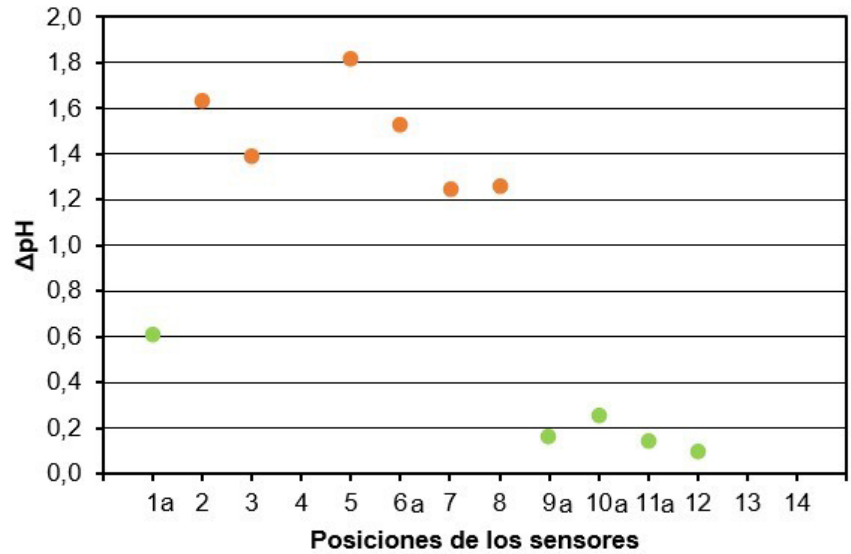

- Nivel de riesgo 1

- Nivel de riesgo 3

vación de las pinturas. De igual modo, en la gráfica de la figura 15 se observa cómo la alteración ocasional del pH del aire a consecuencia de la manipulación del ácido clorhídrico no sobrepasa el nivel bajo de riesgo por choque ácido. Por último, la figura 16 presenta los resultados de las variaciones de la acidez ambiental del interior del taller, debidas a los efectos de la deposición ácida seca que se produjeron en la Comunidad de Madrid durante el mes 
de marzo de 2019. De acuerdo con los criterios anteriormente señalados, la sala $B$ del aula se enmarca dentro del nivel medio de riesgo, mientras que el resto de salas permanecieron dentro del nivel bajo.

\section{CONCLUSIONES}

Con la investigación realizada se ha podido comprobar, por un lado, que los sensores de $\mathrm{pH}$ ambiental tienen una sólida aplicación en el ámbito de la conservación preventiva de bienes culturales. Estos sensores constituyen actualmente el único procedimiento de evaluación capaz de medir el pH del aire, valor resultante de la sinergia de los diferentes contaminantes ambientales cuya presencia supone un factor de riesgo para la preservación de los bienes y para la salud humana. $\mathrm{Y}$, por otro lado, que la conservación preventiva en los talleres de restauración carece de una bibliografía específica, aplicándose las directrices desarrolladas para asegurar la conservación de las obras pictóricas en museos y depósitos, entre otros. Sin embargo, estas medidas no cuentan con la exposición de los bienes a contaminantes derivados del almacenamiento y manipulación de reactivos químicos.

Mediante las evaluaciones de acidez ambiental realizadas se han podido detectar variaciones de $\mathrm{pH}$ debidas a la utilización de reactivos, la afluencia de personal en el taller y el régimen de ventilación, así como por factores macroclimáticos del exterior. De forma general, las oscilaciones de $\mathrm{pH}$ en las salas del taller no suponen un riesgo para la conservación de las obras, salvo aquellos valores registrados durante el fenómeno de deposición seca ácida que tuvo lugar y que supuso unas condiciones ambientales desfavorables para las pinturas presentes en la sala $B$. En condiciones habituales el ambiente del taller de restauración es esencialmente neutro, lo que garantiza la salubridad del personal y la conservación de las obras pictóricas que están en proceso de restauración.

\section{Agradecimientos}

Los autores expresan su agradecimiento a la Dra. Alicia Sánchez Ortiz, profesora titular de la Universidad Complutense de Madrid, por facilitar el taller que ha servido como espacio de estudio experimental para desarrollar la presente investigación, así como al Ldo. Fernando Agua y al Dr. Manuel García Heras, miembros del grupo de investigación CERVITRUM (Cultura Material y Patrimonio) del Instituto de Historia, CSIC, Madrid, por su apoyo y colaboración. También agradecen al Programa JAEIntro del CSIC por la concesión de la beca de iniciación a la investigación en el curso 2018-2019 con la cual se llevó a cabo esta investigación, y al Programa TOP Heritage (ref. S2018/NMT-4372) financiado por la Comunidad de Madrid y Fondo Social Europeo por un contrato de investigador de apoyo. Los autores también agradecen el apoyo profesional de la Red TechnoHeritage de Ciencia y Tecnología para la Conservación del Patrimonio Cultural, y de la Plataforma Temática Interdisciplinar del CSIC Patrimonio Abierto: Investigación y Sociedad (PTI-PAIS). 


\section{BIBLIOGRAFÍA}

- Carmona, N., Villegas, M.A. y Fernández, J.M. (2004) Optical sensors for evaluating environmental acidity in the preventive conservation of historical objects. Sensor Actuat A-Phys, vol. 3, n. ${ }^{\circ} 116$, pp. 398-404

- Cremonesi, P. (2011) L'ambiente acquoso per il trattamento di opere policrome. I Talenti.metodologie, tecniche e formazione nel mondo del restauro. Padova: II Prato

- García, I. (2013) La conservación preventiva de bienes culturales. Madrid: Alianza Forma

- García-Heras, M., Gil, C., Carmona y N. Villegas, M.A. (2005a) Optical behaviour of pH detectors based on solgel technology. Analytica Chimica Acta, vol. 1, n. ${ }^{\circ}$ 540, pp. 147-152

- García-Heras, M., Kromka, K., Faber, J., Karaszkiewicz, P. y Villegas, M.A. (2005b) Evaluation of air acidity through optical sensors. Environmental Science and Technology, vol. 10, n. $^{\circ} 39$, pp. 3743-3747

- García-Heras, M., Villegas, M.A. (2015) Innovación y gestión de la conservación preventiva en museos: un ejemplo con colecciones de vidrio y materiales cerámicos. Revista ph investigación, n. ${ }^{\circ}$ 5, pp. 103-117. Disponible en: https://www.iaph.es/revistaph/index.php/revistaph/article/ view/4060 [Consulta: 17/11/2020]

- Grzywacz, M. (2006) Monitoring for gaseous pollutants in museum environments. Tools for conservation. Los Ángeles: Getty Conservation Institute, pp. 86-90 Disponible en: https:// www.getty.edu/conservation/publications_resources/ pdf publications/pdf/monitoring.pdf [Consulta: 17/11/20 20]

- Llorente-Alonso, A., Peña-Poza, J., De Arcas, G., García-Heras, M., López Navarro, J.M. y Villegas, M.A (2013) Interface electronic system for measuring air acidity with optical sensors. Sensors and Actuators A Physical, n. ${ }^{\circ}$ 194, pp. 67-74

- Llorente-Alonso, A., Peña-Poza, J., Domínguez, J.F. Gil, C., García Heras, M. y Villegas Broncano, M.A. (2013) Evaluation of environmental conditions of the Museo del Ejército (Toledo, Spain) by means of Sol-Gel optical sensors. En: Rogerio-Candelera, M.A., Lazzari, M. y Cano, E. (ed.) Science and Technology for the Conservation of Cultural Heritage. London: CRC Press. Taylor \& Francis Group. Balkema, pp. 15-18

- Medina, M.A. (2019) La contaminación en marzo aumenta el doble en la Comunidad que en la capital. El País, ed. Madrid, 11 de abril de 2019. Disponible en: https:// elpais.com/ccaa/2019/04/09/madrid/1554834524_716445. html [Consulta: 10/08/2019]

- Peña-Poza, J., Conde Moreno, J.F., Agua Martínez, F., García-Heras, M. y Villegas Broncano, M.A. (2013)
Application of sol-gel based sensors to environmental monitoring of Mauméjean stained glass windows housed in two different buildings at downtown Madrid. Boletín de la Sociedad Española de Cerámica y Vidrio, vol. 6, n. ${ }^{\circ} 52$, pp. 268-276

- Peña-Poza,J., Conde Moreno, J.F., Palomar, T., Agua Martínez, F., García-Heras, M. y Villegas, M.A. (2011) Environmental evaluation of the holdings at the CCHSCSIC Tomas Navarro Tomas Library. Revista Española de Documentación Científica, vol. 1, n. ${ }^{\circ}$ 34, pp. 65-78

- Peña Poza, J., Gálvez Farfán, J.M., González Rodrigo, M., García Ramírez, S., Villegas Broncano, M.A. y Garcia Heras, M. (2015) Propuesta de protocolo de valoración de la acidez ambiental en salas y vitrinas de la exposición temporal El último viaje de la fragata Mercedes. La razón frente al expolio (Museo Naval, Madrid). Ge-conservación, n. ${ }^{\circ}$ 8, pp. 4-26. Disponible en: https://www.ge-iic.com/ojs/ index.php/revista/article/view/279 [Consulta: 2/12/2020]

- Peña-Poza, J., García-Heras, M., Palomar, T., Laudy, A., Modzelewska, E. y Villegas, M.A. (2011) Environmental evaluation with chemical sensors in the Palace Museum of Wilanów, Bulletin Polish Academy of Sciences: Technical Sciences, vol. 59, n. ${ }^{\circ} 3$, pp. 247-252

- Peña Poza, J. (2014) Optimización, comportamiento y preindustrialización de sensores ambientales basados en la tecnología Solgel. Tesis doctoral, Universidad Autónoma de Madrid. Disponible en: http://hdl.handle.net/10486/664117 [Consulta: 11/03/2019]

- Plan Nacional de Conservación Preventiva (2011) Madrid: Ministerio de Cultura, Secretaría de Estado de Cultura, 2011

- Prevención de Riesgos Laborales (2019) Administración General del Estado. España

- Salas, C. y Porras, M. (2018) Criterios de intervención en pintura de caballete. Proyecto COREMANS. Madrid: Ministerio de Cultura y Deporte

- Sánchez, A. (2012) Restauración de obras de arte: pintura de caballete. Madrid: Akal

- Villegas Broncano, M.A., García Heras, M., Peña Poza, J., Arcas Castro, G. de, López Navarro, J.M., Barrera López de Turiso, E. y Llorente Alonso, A. (2010) Sistema para la determinación de acidez ambiental y método que hace uso del mismo. Patente española ref. P201031071. Propietarios: CSIC y UPM

- Villegas, M.A., Peña-Poza, J. y Garcia-Heras, M. (2016) Sol-Gel Environmental Sensors for Preventive Conservation of Cultural Heritage. En: Klein, L.C., Jitianu, A. y Aparicio, M. (ed.) Handbook of Sol-Gel Science and Technology, $2 .{ }^{\text {a }}$ edición. Switzerland: Springer International Publishing, pp. $1-32$ 